Felipe da Rocha Lima

\title{
Decisão de Sazonalização para Otimizar Carteira de Contratos com Hidrelétricas e Eólicas no Brasil
}

\section{Dissertação de Mestrado}

Dissertação apresentada como requisito parcial para obtenção do grau de Mestre pelo Programa de PósGraduação em Administração de Empresas do Departamento de Administração de Empresas da PUC-Rio.

Orientador: Prof. Leonardo Lima Gomes Co-orientador: Profa. Marta Corrêa Dalbem 
Felipe da Rocha Lima

\title{
Decisão de Sazonalização para Otimizar Carteira de Contratos com Hidrelétricas e Eólicas no Brasil
}

\begin{abstract}
Dissertação apresentada como requisito parcial para obtenção do grau de Mestre pelo Programa de PósGraduação em Administração de Empresas do Departamento de Administração de Empresas do Centro de Ciências Sociais da PUC-Rio. Aprovada pela Comissão Examinadora abaixo assinada.
\end{abstract}

Prof. Leonardo Lima Gomes

Orientador PUC-Rio

Prof. Leonardo Lima Gomes PUC-Rio

Profa. Marta Corrêa Dalbem UniGranRio

Prof. Luiz Eduardo Teixeira Brandão PUC-Rio

Prof. Carlos de Lamare Bastian Pinto UniGranRio

Prof. Mônica Herz Vice-Decana de Pós-Graduação do CCS

Rio de Janeiro, 03 de abril de 2012 
Todos os direitos reservados. É proibida a reprodução total ou parcial do trabalho sem autorização da universidade, do autor e do orientador.

Felipe da Rocha Lima

Graduou-se em Engenharia de Telecomunicações na Universidade Federal Fluminense (UFF) em 2006. Em 2010 ingressou no Mestrado em Administração de Empresas da PUC-Rio. Trabalha na área de operação e manutenção de sistemas de telecomunicações do setor de Tecnologia da Informação e Telecomunicações da Petróleo Brasileiro S.A. - Petrobras.

Ficha Catalográfica

Lima, Felipe da Rocha

Decisão de sazonalização para otimizar carteira de contratos com hidrelétricas e eólicas no Brasil / Felipe da Rocha Lima ; orientador: Leonardo Lima Gomes. 2012.

57 f. : il. (color.) ; $30 \mathrm{~cm}$

Dissertação (mestrado)-Pontifícia Universidade Católica do Rio de Janeiro, Departamento de Administração, 2012.

Inclui bibliografia

1. Administração - Teses. 2. Otimização de carteira. 3. Energia eólica. 4. Medida ômega. 5. Sazonalização. I. Gomes, Leonardo Lima. II. Pontifícia Universidade Católica do Rio de Janeiro. Departamento de Administração. III. Título.

CDD: 658 
Aos meus pais que sempre me incentivaram a buscar meus objetivos e que mostraram a importância de cultivar bons valores e de ter fé. 


\section{Agradecimentos}

Agradeço a Deus, pelas oportunidades que tenho na vida, pela família acolhedora que possuo, por sempre colocar pessoas boas no meu caminho e por me dar força para superar os desafios do dia-a-dia.

Aos meus pais, Maria Cristina de L. da Rocha Lima e Gilberto Fernando da Rocha Lima, pelo suporte e incentivo ao longo deste período. Além de representarem um porto seguro nos momentos de dificuldade e proporcionarem um ambiente familiar de carinho e alegria.

Aos meus irmãos, Fábio da Rocha Lima e Amanda da Rocha Lima, pela compreensão nos momentos em que precisei de apoio e pela boa convivência que nós temos.

À minha namorada Camila Ávila, pelo apoio, incentivo e compreensão quando tive que abdicar de momentos de lazer para me dedicar ao estudo.

Ao meu orientador, professor Leonardo Lima Gomes, pelo tratamento cordial, pela dedicação e por estar sempre disponível para dar os direcionamentos necessários.

À minha co-orientadora, professora Marta Dalbem, pela atenção, por estar sempre pronta a ajudar e pelo suporte em momentos fundamentais do desenvolvimento desta dissertação.

Aos meus colegas de mestrado no IAG, principalmente a Tatiana Pitta, a Viviane Gomes e o Bruno Leite, com quem fiz diversos trabalhos em grupo e, mesmo em situações de estresse, passei momentos de alegria.

À PUC-Rio e ao IAG, pela estrutura, pelo ambiente propício ao desenvolvimento do conhecimento e por oferecer um curso de mestrado acadêmico em horário que permitiu a conciliação com minha rotina de trabalho. 


\section{Resumo}

Lima, Felipe da Rocha; Gomes, Leonardo Lima. Decisão de Sazonalização para Otimizar Carteira de Contratos com Hidrelétricas e Eólicas no Brasil. Rio de Janeiro, 2012. 57p. Dissertação de Mestrado Departamento de Administração, Pontifícia Universidade Católica do Rio de Janeiro.

A Sazonalização é o processo de dividir as quantidades anuais de um contrato de fornecimento de energia elétrica em montantes mensais. Trata-se de flexibilidade contratual facultativa geralmente determinada ou imposta pelo agente comprador. A participação das usinas eólicas na matriz energética brasileira tem aumentado nos últimos anos, sendo a energia dos ventos uma fonte renovável que apresenta uma característica interessante de complementaridade em relação à vazão das usinas hidrelétricas. As carteiras de empresas comercializadoras passam então a abrigar contratos com usinas eólicas e hidrelétricas. Tendo como meta auferir maiores ganhos algumas comercializadoras realizam operações descasadas e podem utilizar a flexibilidade da sazonalização de contratos. Neste trabalho foi tomada como referência uma comercializadora que possui uma carteira composta de um contrato de venda de um montante mensal fixo de energia, um contrato de compra da energia elétrica de uma usina eólica e um contrato de compra de energia de uma hidrelétrica que pode ser sazonalizado. Foi desenvolvida uma metodologia para determinar o perfil de sazonalização ideal a ser utilizado para aumentar os lucros da carteira de contratos. A maximização da medida Ômega $(\Omega)$ foi a métrica utilizada para analisar os cenários e foram consideradas restrições de Value at Risk - VaR nas análises realizadas. Os resultados encontrados mostram que a maximização da medida $\Omega$ é obtida concentrando-se toda compra de energia da hidrelétrica no segundo semestre do ano. Entretanto, à medida que a restrição de $\mathrm{VaR}$ torna-se mais severa diminuindo o grau de liberdade para realizar a sazonalização a distribuição dos montantes de energia elétrica aproxima-se da uniformidade entre os meses do ano. 


\section{Palavras-chave}

Otimização de Carteira; Energia Eólica; Medida Ômega; Sazonalização 


\section{Abstract}

Lima, Felipe da Rocha; Gomes, Leonardo Lima (Advisor). Decision of Seasonal Adjustments to Optimize Portfolio of Contracts with Hydroelectrics and Wind Farms in Brazil. Rio de Janeiro, 2012. 57p. MSc. Dissertation - Departamento de Administração, Pontifícia Universidade Católica do Rio de Janeiro.

The seasonal adjustment is the process of dividing the annual quantities of an electricity supply contract in monthly amounts. This is a contractual flexibility generally determined or imposed by the contract buyer. The share of wind farms in the Brazilian energy system has increased in the last few years and wind energy is a renewable source that presents an interesting feature of complementarity with hydroelectric power plants in Brazil. Therefore, contracts with wind farms and hydroelectric plants are becoming part of the trading companies' portfolios. Aiming to reach higher gains, some energy traders enter into uncovered trading positions and use the flexibility of seasonal adjustments of contracts to combine these two sources in a portfolio. This study used as reference a trading company that has a portfolio composed of a sales contract for a fixed monthly amount of energy, a contract to buy electricity from a wind farm and a contract to purchase electricity from a hydroelectric plant that can be seasonally adjusted. A methodology is proposed to find the ideal profile of seasonal adjustments that should be used to increase the portfolio's profits. The maximization of the Omega measure $(\Omega)$ - along with Value at Risk (VaR) restrictions-, was the metric used to analyze the scenarios and choose the optimal portfolio. The results found show that the maximization of the $\Omega$ measure is obtained by concentrating all the energy bought from the hydroelectric in the second semester of the year. However, as the constraint VaR becomes more severe restricting the freedom to make seasonal adjustments, the distribution of energy amounts between the months of the year approaches the uniformity.

\section{Keywords}

Portfolio Optimization; Wind Energy; Omega Measure; Seasonal Adjustments 


\section{Sumário}

1 Introdução 14

2 Referencial Teórico 18

2.1. Medidas de Desempenho de Carteiras $\quad 18$

2.2. Medida Ômega 19

3 Mercado Brasileiro de Energia Elétrica 24

3.1. Setor Elétrico Brasileiro 24

3.2. Características dos Contratos Bilaterais 25

3.3. Sazonalização 26

3.4. Preço de Liquidação das Diferenças 28

3.4.1. Conceitualização do PLD 28

3.4.2. Cálculo do PLD 29

3.4.3. Simulação dos PLDs 30

4 Desenvolvimento do Modelo 31

4.1. Carteira de Contratos 31

4.2. Simulação dos Ventos 34

4.3. Simulação dos PLDs $\quad 37$

4.4. Otimização 38

5 Resultados $\quad 40$

5.1. Valores da Medida $\Omega$ e de $V R_{95 \%}$ para Diferentes Metas (L) nos Casos de Sazonalização Ótima 40

5.2. Variação da Medida $\Omega$ sem Restrição de $\operatorname{VaR}_{95 \%}$, Quando se Adota a Sazonalização Ótima 41

5.3. Variação da Medida $\Omega$ sem Restrição de $\operatorname{VaR}_{95 \%}$ Considerando apenas Contratos de Compra de Energia Eólica 42

5.4. Valores da Medida $\Omega$ Fixando o $\operatorname{VaR}_{95 \%}$ 
5.5. Sensibilidade da Medida $\Omega$ aos Preços Contratados

5.6. Decisão de Sazonalização

6 Conclusões

7 Referências Bibliográficas 


\section{Lista de figuras}

Figura 1 - llustração do Cálculo da Medida Ômega. 20

Figura 2 - Exemplo de distribuição de retornos com $L=1,4$. 21

Figura 3 - FDP Weibull para o mês de agosto. 36

Figura 4 - Curva de Potência do Aerogerador. 36

Figura 5 - Potências médias mensais estimadas. 37

Figura 6 - PLD mensal para 2012.

Figura 7 - Ômega e VaR $95 \%$ variando meta. $\quad 41$

Figura 8 - Ômega em relação à sazonalização. 42

Figura 9 - Ômega para contratos com duas usinas eólicas 43

Figura 10 - Ômega restrito por $\mathrm{VaR}_{95 \%}$. 44

Figura 11 - Sensibilidade de $\Omega$ aos preços contratuais. $\quad 46$

Figura 12 - Perfil de Sazonalização sem VaR. 47

Figura 13 - Perfis de Sazonalização e PLDs médios. 48

Figura 14 - Perfis de Sazonalização com VaR95\% de R $\$ 1,2$ milhão. $\quad 49$

Figura 15 - Perfis de Sazonalização com VaR95\% de $R \$ 1,7$ milhão. $\quad 50$

Figura 16 - Variação de Metas de $\operatorname{VaR}_{95 \%}$. 51 


\section{Lista de tabelas}

Tabela 1 - Retornos e suas probabilidades. 22

Tabela 2 - Diferenças entre retornos e nível $L$. 22

Tabela 3 - Resumo de informações contratuais. 34

Tabela 4 - Estatística Descritiva PLD 2012.

Tabela 5 - Variação de preços contratados. 


\section{Lista de Siglas e Abreviaturas}

$\mathrm{ACL}$ - Ambiente de Contratação Livre

ACR - Ambiente de Contratação Regulada

ANEEL - Agência Nacional de Energia Elétrica

BEN - Balanço Energético Nacional

CCEAL - Contratos de Compra de Energia no Ambiente Livre

CCEAR - Contratos de Comercialização de Energia no Ambiente

Regulado

CCEE - Câmara de Comercialização de Energia Elétrica

CEPEL - Cento de Pesquisas de Energia Elétrica

CMO - Custos Marginais de Operação

COFINS - Contribuição para o Financiamento da Seguridade Social

CSSL - Contribuição Social Sobre o Lucro Líquido

EPE - Empresa de Pesquisa Energética

IRPJ - Imposto de Renda Pessoa Jurídica

MAE - Mercado Atacadista de Energia Elétrica

MME - Ministério de Minas e Energia

ONS - Operador Nacional do Sistema

$\mathrm{PCH}$ - Pequena Central Hidrelétrica

PDEE - Plano Decenal de Expansão de Energia

PIS - Programa de Integração Social

PLD - Preço de Liquidação das Diferenças

PROINFA - Programa de Incentivo a Fontes Alternativas

SEB - Setor Elétrico Brasileiro

SIN - Sistema Interligado Nacional

SMC - Simulação de Monte Carlo

VaR, V@R - Value At Risk 